\title{
Persistence of Left Superior Vena Cava in Nonagenarian Undergoing Pacemaker Implant: Case Report
}

\author{
Lucas Hollanda Oliveira ${ }^{1,2,3,{ }^{*},}$, Christian Moreno Luize ${ }^{1,2}$, Ricardo Sobral de Carvalho ${ }^{1,2}$, Marcel \\ Fernando Silva Carvalho ${ }^{1,2}$, Danusa Moreira Lago ${ }^{1}$, Cristiano de Oliveira Dietrich ${ }^{3,4}$, Claudio Cirenza ${ }^{3}$
}

\section{ORCID IDs}

Oliveira LH (D) https://orcid.org/0000-0002-6770-4462

Luize CM (D) https://orcid.org/0000-0002-4024-1963

Carvalho RS (D) https://orcid.org/0000-0002-5349-4265

\author{
Carvalho MFS (D) https://orcid.org/0000-0002-0559-0375 \\ Lago DM (D) https://orcid.org/0000-0003-2144-0454 \\ Dietrich CO (D) https://orcid.org/0000-0002-7373-9119 \\ Cirenza C (D) https://orcid.org/0000-0001-7290-9158
}

\begin{abstract}
Introduction: The persistence of the left superior vena cava (PLSVC) is a congenital malformation of the veins that drain blood from the upper body, causing the flow to the right atrium to be processed through the coronary sinus. Case report: We describe the case of a nonagenarian woman, previously asymptomatic until she developed total atrioventricular block when she was referred for implantation of a double chamber pacemaker. Persistence of the left superior vena cava was detected during the procedure and confirmed by venography. The implantation of the electrodes was performed safely and without complications. Conclusion: Despite not usually causing symptoms, the presence of PLSVC can make invasive procedures a challenging task. Knowledge of anatomy and the use of simple techniques can facilitate interventions in this scenario.
\end{abstract}

KEYWORDS: Artificial pacemaker; Superior vena cava; Embryology; Congenital abnormalities; Coronary sinus.

\section{INTRODUCTION}

Persistence of the left superior vena cava (PLSVC) is a congenital malformation of the veins that drain blood from the upper body, causing the flow to the right atrium to proceed through the coronary sinus ${ }^{1}$. Its prevalence is little known, as it rarely causes symptoms, is estimated between 0.2 and $3 \%$ of the general population ${ }^{1,2}$ and around $0.47 \%$ among individuals referred for implantation of cardiac devices ${ }^{3}$.

The primitive heart-formed at the end of the fourth week comprises the venous sinus, the primitive ventricle, the cardiac bulb and the arterial trunk. Blood drainage to the venous sinus occurs through three major systems: the vitelline

1. Fundação Bahiana de Cardiologia - Salvador (BA), Brazil.

2. Hospital São Rafael - Salvador (BA), Brazil.

3. Escola Paulista de Medicina - São Paulo (SP), Brazil.

4. Hospital Moriah - São Paulo (SP), Brazil.

*Corresponding author: lucas.hollanda78@icloud.com

Received: Apr 23, 2021 | Accepted: May 13, 2021 
vein system, which drains blood from the vitelline sac; the umbilical vein system, which carries blood from the placenta; and the cardinal vein system, which is intraembryonary. Around the eighth to tenth week, the caudal portion of the upper right cardinal vein originates the superior vena cava. The left vitelline vein atrophies, while the right one participates in the formation of the inferior vena cava. Under normal conditions, the cranial portions of the upper right and left cardinal veins merge, forming the brachiocephalic veins. The caudal portion of the left cardinal vein atrophies and invaginates within Marshall's ligament, giving rise to the coronary sinus, which in turn flows into the right atrium. Under certain littleunderstood stimuli, the atrophy of the common left cardinal vein does not occur, giving rise to $\mathrm{PLSVC}^{1,2}$. Innominate veins may not degenerate, and, depending on this phenomenon, anatomical variations may arise 4 .

In approximately $90 \%$ of PLSVC, venous drainage on the right is preserved through the superior vena cava, a condition known as double superior vena cava (DSVC). In $65 \%$ of these cases, the vena cava travel in parallel, one on each side of the mediastinum, with no communication between them. In the others, a brachiocephalic vein can communicate between the vessels (bridging vein) ${ }^{1}$. Bearing in mind that 80 to $90 \%$ of the time, the left upper vena cava flows into the right atrium through the coronary sinus, no hemodynamic repercussions are usually observed. When this does not occur, venous blood drains into the left atrium, leading to a shunt from right to left ${ }^{4}$.

\section{CASE REPORT}

A 91-year-old hypertensive patient, investigated on an outpatient basis for dizziness and malaise, was hospitalized for worsening symptoms and adynamia in the last two weeks. She was taking losartan $50 \mathrm{mg} /$ day and amlodipine $5 \mathrm{mg} / \mathrm{day}$. An intraventricular conduction abnormality and a 2:1 second-degree atrioventricular block were observed in a 24-hour Holter monitoring (Fig. 1a). A transthoracic echocardiogram performed on the day before showed a preserved biventricular function, mild myocardial hypertrophy and type I diastolic dysfunction. The admission 12-lead electrocardiogram showed a total atrioventricular block (TAB) with a wide QRS escape rhythm (Fig. 1b). Given the absence of reversible factors leading to the TAB and following specialty guidelines, a double-chamber pacemaker was indicated ${ }^{5}$.

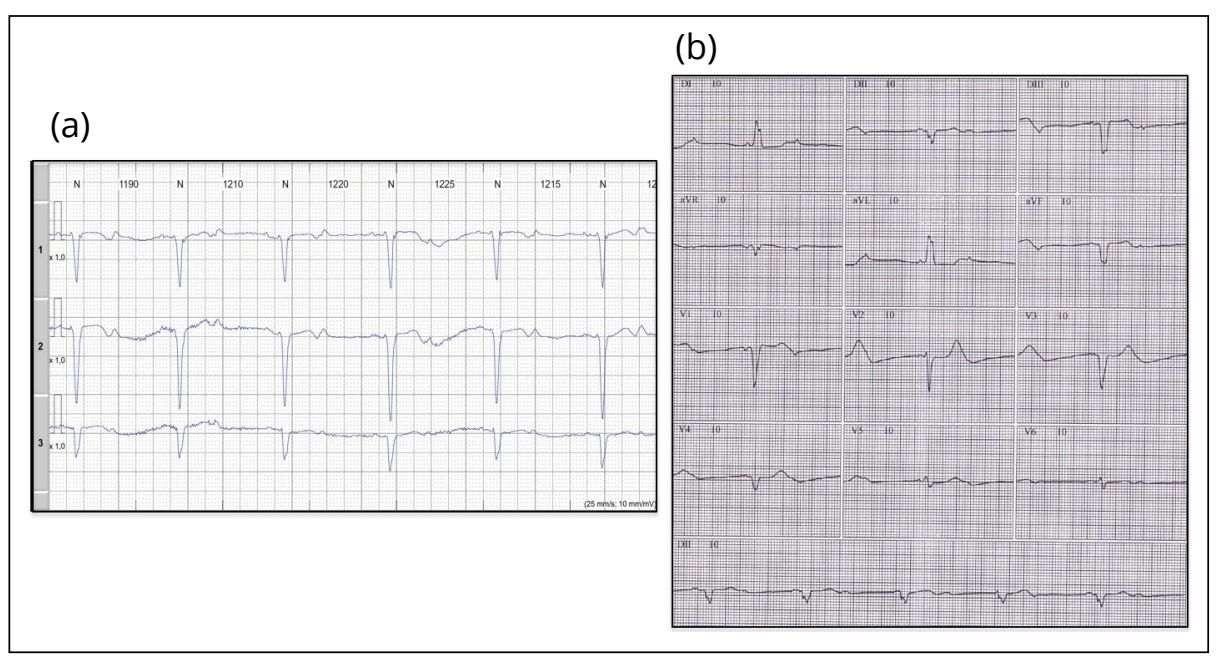

Figure 1. (a) Intraventricular conduction abnormality and 2:1 second degree atrioventricular block in a 24-hour Holter monitoring. (b) A 12-lead electrocardiogram with atrioventricular dissociation and wide QRS escape: total atrioventricular block.

The left hemithorax was prepared aseptically, and the left cephalic vein was dissected. Subsequently, a subcutaneous pocket was created. After phlebotomy, a $0.35 \mathrm{~mm}$ guidewire was advanced through the cephalic vein, and its progression assumed an anomalous course, running through the subclavian vein, passing through the mediastinum to the right lateral border of the cardiac silhouette (Fig. 2). 


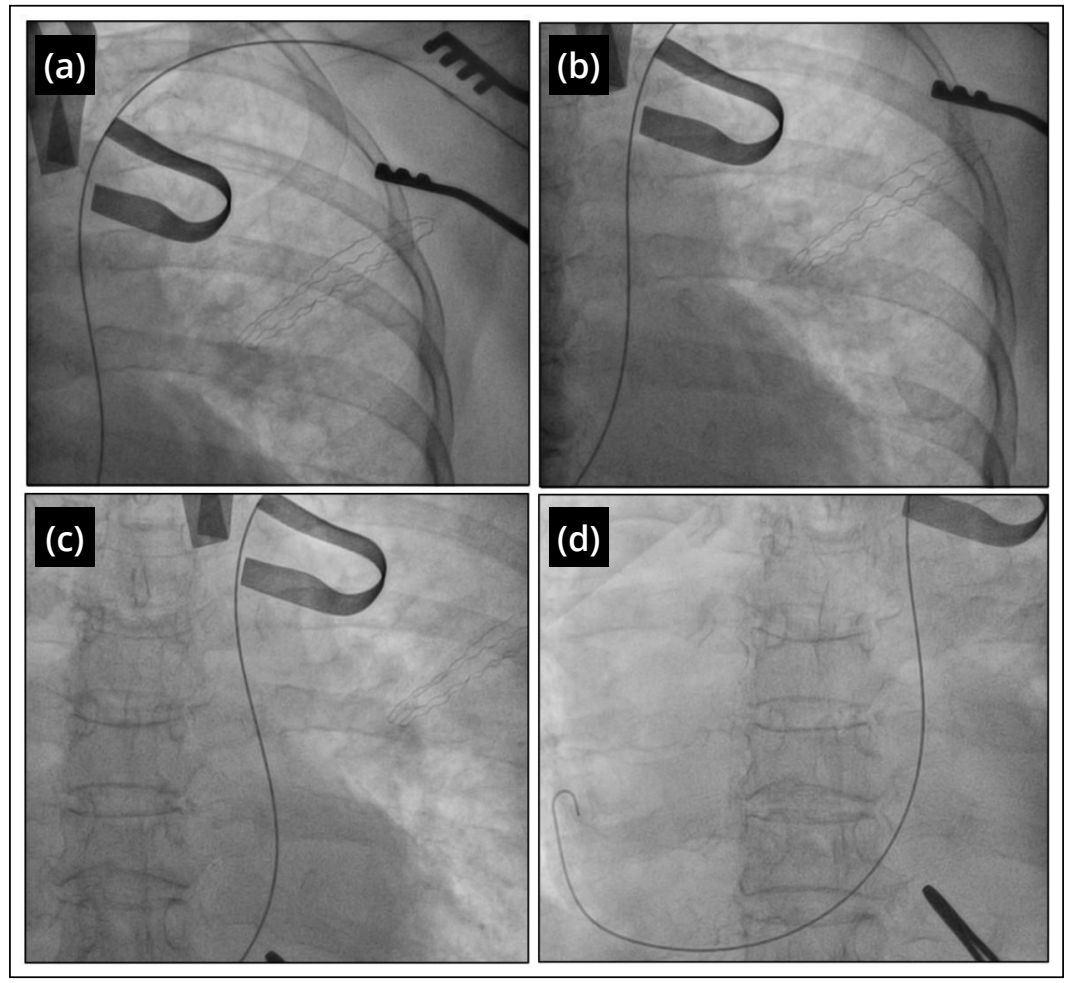

Figure 2. The $0.035 \mathrm{~mm}$ guide wire pathway on fluoroscopy (a) from the lateral border of the left cephalic vein, (b and c) passing through the mediastinum (d) to the right lateral border of the cardiac silhouette.

In order to better understand the anatomy, a $6 \mathrm{~F}$ introducer was placed inside the subclavian vein over the guidewire, and venography was performed (Fig. 3). The design of the vessels was compatible with the persistence of the left superior vena cava. With the aid of fluoroscopy, the left axillary vein was punctured and a second guidewire was placed inside the vessel.

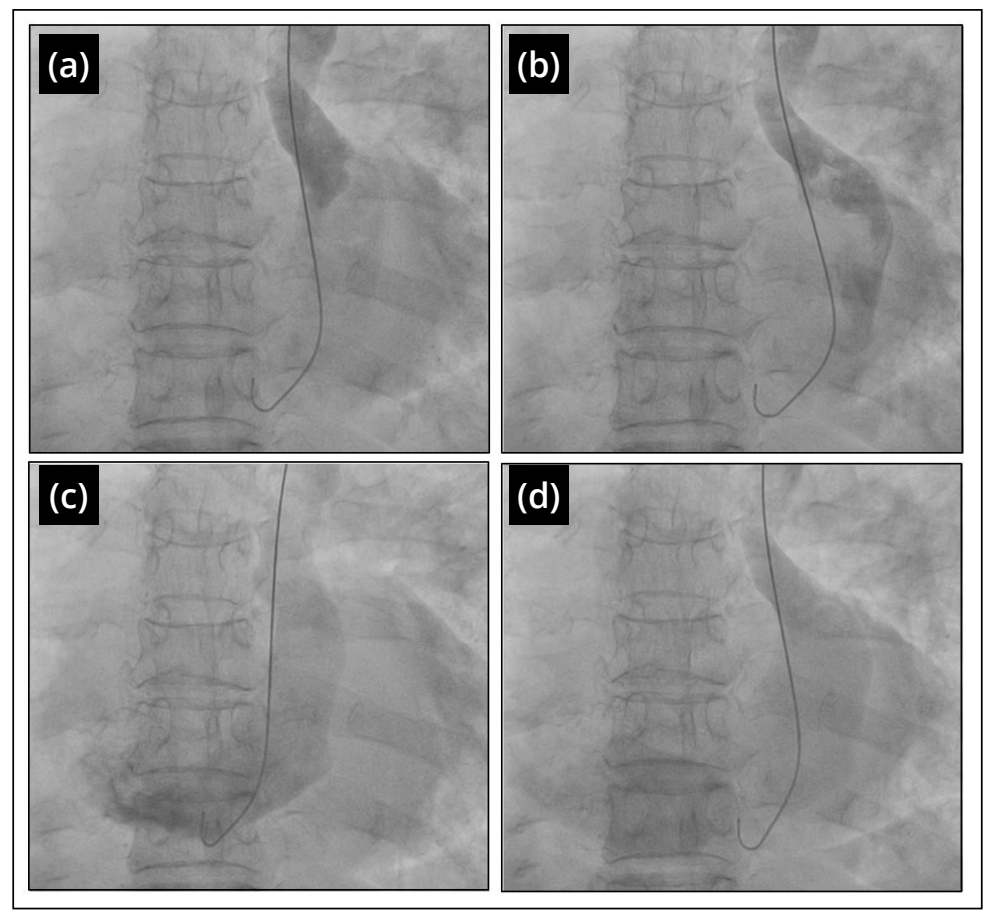

Figure 3. Sequential images of angiography through the left cephalic vein showing (a) the end of the left innominate vein and (b) the beginning of the dilated coronary sinus (c) opening into the right atrium. (d) A left venous drainage overview, from the innominate vein, through the coronary sinus, right atrium to the right ventricle. 
A Biotronik Solia MRI $60 \mathrm{~cm}$ ventricular electrode was progressed through a $6 \mathrm{~F}$ introducer to a region close to the lateral wall of the right atrium. In that place, a J- shaped guide was introduced inside the electrode and its convex face was lightly pressed against the lateral wall of the right atrium. In a $30^{\circ}$ right lateral oblique view, using smooth and simultaneous movements of advancing the electrode and retracting the guide, the electrode was moved into the right ventricle. Using a $30^{\circ}$ left lateral oblique view , the electrode was positioned at the interventricular septum with a second pre-shaped guide (Fig. 4). Over the second $0.035 \mathrm{~mm}$ guidewire, a second $6 \mathrm{~F}$ introducer was advanced into the vessel, and a Biotronik Solia MRI $53 \mathrm{~cm}$ atrial electrode was actively attached to the middle lateral wall of the right atrium (Fig. 4).

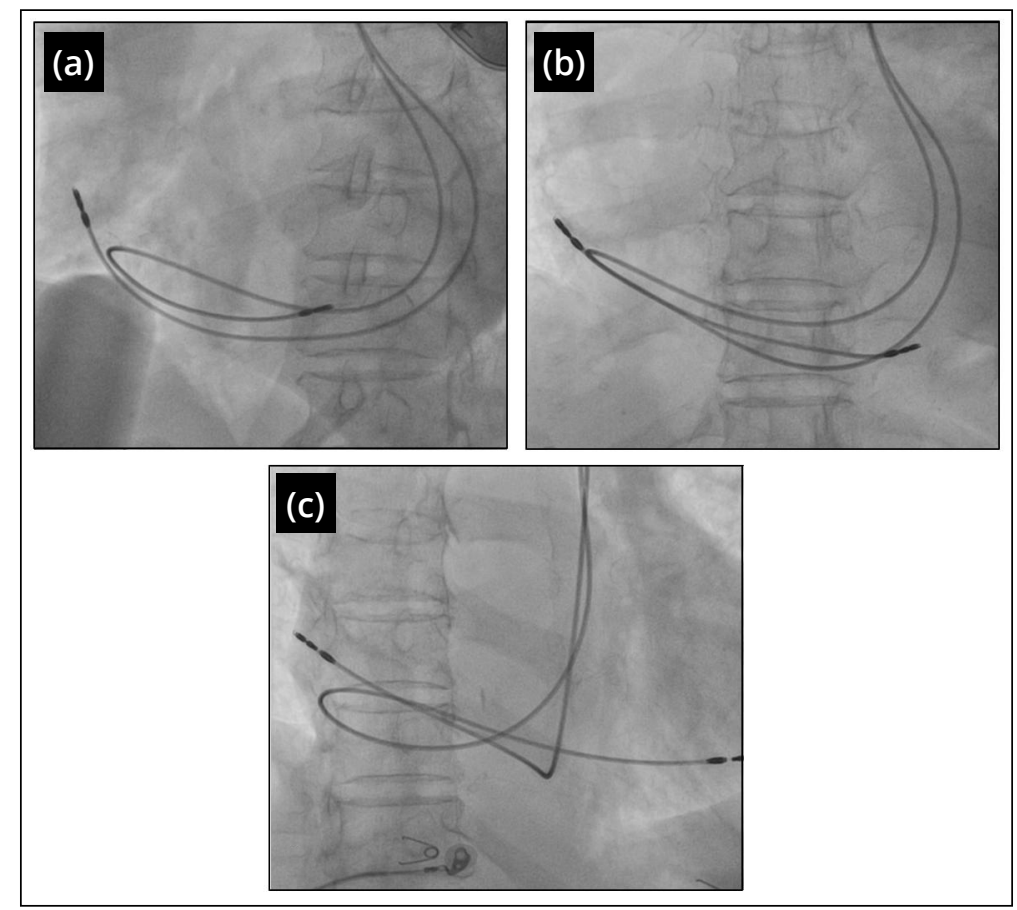

Figure 4. (a) $30^{\circ}$ Left anterior oblique view, (b) anteroposterior view and (c) $30^{\circ}$ right anterior oblique view of the final position of atrial(middle portion of the right atrium) and ventricular (tip facing the septum) electrodes

\section{DISCUSSION}

PLSVC is a rare congenital anomaly of the vessels that drain blood to the chest, rarely causing hemodynamic repercussions and symptoms. Its importance, however, is notable during invasive procedures, in which unusual anatomical changes can transform usually simple interventions into complex and challenging situations. Prior knowledge of the anomaly, in theory, would assist in planning the intervention. Given the rarity of PLSVC, an eventual screening strategy would not be cost-effective.

The suspicion or diagnosis of PLSVC, in general, is made incidentally by imaging methods (angiotomography, magnetic resonance and echocardiogram) used in the investigative process of other diseases. Since transthoracic echocardiography is a tool commonly used to assess ventricular function and define the method of ventricular stimulation, special attention should be given to the signs of PLSVC. Coronary sinus dilation and suggestive venous path after bullous contrast injection in the left upper limb are signs of malformation ${ }^{2,6}$. In the vast majority of cases, however, the anomaly is discovered during invasive venography procedures.

We present the case of a nonagenarian with PLSVC who underwent successful implantation of a double chamber pacemaker. The absence of symptoms related to venous anomaly until the tenth decade of life illustrates the fortuitous of PLSVC. Understanding anatomy, associated with using techniques to overcome path angulations imposed by congenital changes, makes the procedure simple and has allowed more complex interventions such as multisite pacemaker implantation ${ }^{7}$. 
Other diagnostic possibilities should be ruled out as it is a rare anomaly, including inadvertent arterial puncture. The path of the guidewire into the left ventricle resembles that of PLSVC and can confuse the interventionist. In these cases, the guidewire does not reach the right end of the cardiac silhouette, and the red and pulsating aspect of the blood corroborates the positioning in the arterial bed. Additionally, the cephalic vein dissection technique can ensure that the vascular path is venous territory. The positioning of the guidewire in the pericardiophrenic vein, located on the lateral edge of the mediastinum and responsible for venous drainage from the pericardium and diaphragm, can be a confusing factor. Its connection with the internal thoracic vein, upper intercostal or left brachiocephalic should be analyzed so that the differential diagnosis is carried out correctly.

Furthermore, the positioning of the guide in the upper left intercostal vein (responsible for draining blood from the second, third and fourth left intercostal veins into the upper right vena cava by the azygos system) should be excluded. Although rare, positioning in an aberrant left brachiocephalic vein (started after the junction of the left internal jugular and subclavian veins, going inferiorly along the left edge of the mediastinum and connecting back to the brachiocephalic trunk) must also be discarded ${ }^{1}$.

\section{CONCLUSION}

Despite not usually causing symptoms, the presence of PLSVC can make invasive procedures a challenging task. Knowledge of anatomy and simple techniques such as bending the electrode to enter the right ventricle can facilitate interventions in these scenarios.

\section{FUNDING}

Not applicable.

\section{AUTHOR'S CONTRIBUTION}

Conceptualization and Manuscript writing: Oliveira, LH; Metodology: Luize, CM, Carvalho RS, Carvalho MFS; Critical revision: Dietrich CO, Cirenza C, Lago, DM.

\section{DATA AVAILABILITY STATEMENT}

The datasets generated during and/or analyzed during the current study are available from the corresponding author on reasonable request.

\section{REFERENCES}

1. Azizova A, Onder O, Arslan S, Ardali S, Hazirolan T. Persistent left superior vena cava: clinical importance and differential diagnoses. Insights Imaging. 2020;11:110. https://doi.org/10.1186\%2Fs13244-020-00906-2

2. Rigatelli G. Congenitally persistent left superior vena cava: A possible unpleasant problem during invasive procedures. J Cardiovasc Med. 2007;8(7):483-7. https://doi.org/10.2459/01.jcm.0000278448.89365.55

3. Morgan DR, Hanratty CG, Dixon LJ, Trimble M, O'Keeffe DB. Anomalies of cardiac venous drainage associated with abnormalities of cardiac conduction system. Europace. 2002;4(3):281-7. https://doi.org/10.1053/eupc.2002.0248 
4. Goyal SK, Punnam SR, Verma G, Ruberg FL. Persistent left superior vena cava: A case report and review of literature. Cardiovasc Ultrasound. 2008;6:50. https://doi.org/10.1186\%2F1476-7120-6-50

5. Kusumoto FM, Schoenfeld MH, Barrett C, Edgerton JR, Ellenbogen KA, Gold MR, et al. 2018 ACC/AHA/HRS Guideline on the Evaluation and Management of Patients With Bradycardia and Cardiac Conduction Delay: A Report of the American College of Cardiology/ American Heart Association Task Force on Clinical Practice Guidelines and the Heart Rhythm. J AM Coll Cardiol. 2019;74(7):932-87. https://doi.org/10.1016/j.jacc.2018.10.043

6. Batouty NM, Sobh DM, Gadelhak B, Sobh HM, Mahmoud W, Tawfik AM. Left superior vena cava: cross-sectional imaging overview. Radiol Medica. 2020;125(3):237-46. https://doi.org/10.1007/s11547-019-01114-9

7. Hollanda L, Sobral R, Luize C, Carvalho W, Carvalho M, Passos L. Cardiac Resynchronization Therapy in a Chagasic Patient With Persistent Left Superior Vena Cava. JACC Case Rep. 2019;1(3):387-90. https://doi.org/10.1016/j.jaccas.2019.07.035 